OPEN ACCESS

Edited by:

Jun Wang,

University of Wisconsin-Madison,

United States

Reviewed by:

Muhammad Saeed,

East China University of Technology,

China

Luteng Zhang,

Chongqing University, China

Chenglong Wang,

Xi'an Jiaotong University, China Yuhao Zhang,

North China Electric Power University,

China

${ }^{*}$ Correspondence:

Xinli Gao

laborforce@163.com

Chunming Zhang

zhangchunming@chinansc.cn

Specialty section:

This article was submitted to

Nuclear Energy,

a section of the journal

Frontiers in Energy Research

Received: 29 September 2021 Accepted: 16 November 2021

Published: 24 December 2021

Citation:

Gao X, Jing J, Han X, Jia B, Tian X, Liu $F$ and Zhang $C$ (2021) Status and Assessment Method of Nuclear Safety

Analysis Software in China.

Front. Energy Res. 9:785494.

doi: 10.3389/fenrg.2021.785494

\section{Status and Assessment Method of Nuclear Safety Analysis Software in China}

\author{
Xinli Gao ${ }^{1,2 *}$, Jianping Jing ${ }^{1,2}$, Xiangzhen Han ${ }^{1,2}$, Bin Jia ${ }^{1,2}$, Xinlu Tian ${ }^{1,2}$, Fudong Liu ${ }^{1,2}$ and \\ Chunming Zhang ${ }^{1 *}$
}

${ }^{1}$ Nuclear and Radiation Safety Center, Ministry of Environmental Protection, Beijing, China, ${ }^{2}$ State Environmental Protection Key Laboratory of Nuclear and Radiation Safety Regulatory Simulation and Validation, Beijing, China

In recent years, China's nuclear power industry has enjoyed a good momentum of development, and related companies have also developed many nuclear analysis software applications. However, as the National Nuclear Safety Administration (NNSA, Chinese nuclear regulatory institution) did not approve any software before 2018 , all these software applications were not evaluated formally, so they have not yet been used in reactor safety analysis. In order to solve this problem, in 2018, the National Nuclear Safety Administration started to carry out an engineering applicability evaluation for software developed by Chinese companies. After several years of review, as the first approved Chinese domestic software, core physics analysis software PCM developed by the China General Nuclear Power Group officially passed the software safety evaluation of the China Nuclear Safety Administration. This study will present the basic situation of the development of China's nuclear power engineering software and introduce the framework, methods, procedures, requirements, and other aspects of China's software safety evaluation work. The evaluation process and evaluation key issues of PCM software will also be illustrated.

Keywords: software assessment, Chinese domestic software, code review procedure, software development progress, PCM

\section{INTRODUCTION}

As of September 2021, there are 62 nuclear reactors in China in total, and 51 of them are under operation. Before Hualong One was built, with the exception of Qinshan unit 2\#, all operating power plants housed imported reactors designed by Canada, Russia, or France. Because of this, the safety analysis software for nuclear power plants used in China was also developed by Canada, Russia, or France, simultaneously. Since such software was reviewed by foreign nuclear safety authorities, NNSA's position was to approve the evaluation opinions of foreign safety review agencies and allow the use of these supporting software applications in the safety analysis and design of domestic nuclear power plants. For example, safety analysis codes WCOBRA/TRAC used in AP1000 safety analysis have been used in AP600, and they have passed NRC's licensing review (USNRC, 2021). Therefore, NNSA has directly approved using WCOBRA/TRAC in AP1000 safety analysis.

As the designs of CAP1400 and AP1000 have similar characteristics in many aspects, the safety analysis software of AP1000 is also used in the design and safety analysis of CAP1400. When NNSA was carrying out the license review of CAP1400, in addition to the ordinary safety analysis report 
review, a review of the applicability of the Westinghouse codes (LOFTRAN, NOTRUMP, WCOBRA/TRAC, and WGOTHIC) to CAP1400 was added.

Nowadays, more and more Chinese nuclear energy companies have independently researched and developed third-generation pressurized water reactors. Since 2011, China General Nuclear Power and the China National Nuclear Corporation have developed two types of third-generation reactors, CPR1000 (Brief, 2006) and ACP1000, respectively. Both are three-loop designs, but the core design is different. These two reactors were both designed with a 60-year lifespan, with 1150 MWe power output, and used a combination of passive and active safety systems. The first constructed units of Hualong One were Fuqing 5 and 6 , followed by Fangjiashan 3 and 4 and Fangchenggang 3 and 4 . Other than the pressurized water reactor, the Shidaowan high-temperature gas-cooled reactor (HTGR) (Zhang et al., 2006); (Zhang et al., 2016) reached the critical stage of success in 2021, and the Xiapu fast reactor demonstration project started construction in 2020 (Zhang, 2016).

For this new reactor design, although various companies have developed a large number of codes to meet the needs of related reactor-type safety analysis, these software applications have not been evaluated and licensed for engineering applications by authoritative organizations, so they cannot be formally applied in in-progress autonomous reactor-type designs. Therefore, evaluating these autonomous safety analysis software applications has become an urgent problem for NNSA to solve.

\section{SOFTWARE DEVELOPMENT PROGRESS IN CHINA}

Now, in China, there are a number of software applications under development, and also, many software applications have been developed and published, such as a software package called COSINE (COre and System Integrated Engine for design and analysis) (Ge et al., 2016); (Hu et al., 2017); (Yu et al., 2013); (Hao, 2015); (Jun et al., 2017); (Aning et al., 2019), published by the State Power Investment Corporation and NESTOR published by the Nuclear Power Institute of China (NPIC) (Dong et al., 2017). Different software applications have been developed for different reactors, for example, the Shanghai Nuclear Engineering Design and Research Institute has developed a series of codes to evaluate the performance of CAP1400, and the China General Nuclear Power Corporation (CGN) has developed specialized safety analysis software for Hualong One safety analysis (Ning et al., 2011). In addition, the China Institute of Atomic Energy (CIAE) has developed its own safety analysis software for sodium-cooled reactor safety analysis (Yang, 2010) (Zhang, 1997).

The NESTOR package includes design, manufacturing, installation, testing, and operation of a nuclear power project based on our Hualong One design (Liu, 2017). It will pave the way for China to export entire nuclear power projects and technologies. The software package consists of 68 pieces of software which will help with more efficient reactor design, safety analysis, live tests, nuclear refueling, and emergency response systems for a plant based on the Hualong One design.

COSINE is the software series for NPP design and analysis. COSINE covers a wide range of engineering functions, including nuclear reactor core physical design, thermal hydraulics design, deterministic accident analysis, radiation-shielding design, fuel design, severe accident analysis, probabilistic safety analysis, and neutron group-constant. COSINE includes 15 programs sorted in 8 software categories, which cover over 80 traditional nuclear power software functions.

The China General Nuclear Power Corporation has developed a series of software applications, including a nuclear fuel assembly calculation code known as PINE (Wang et al., 2018), a 3D core calculation code called COCO (Cai et al., 2016), a system thermalhydraulic analysis code known as GINKGO, and a sub-channel analysis code called LINDEN, and so on (Ke et al., 2019).

Universities in China have also developed many software applications. For example, the research group NuThel from Xi'an Jiaotong University has developed a series of thermalhydraulic analysis software programs (Jie et al., 2013); (Shiying et al., 2018); (Tian et al., 2007); (Xiao et al., 2014), and the NECP team, also from Xi'an Jiaotong University, has developed a complete set of physical safety analysis software programs (Xu et al., 2017); (Hongchun et al., 2019); however, these pieces of software have not been developed for safety analysis of nuclear power plants, are short of complete quality assurance, and have not been certified and validated sufficiently. Therefore, at this stage, NNSA will not evaluate these programs.

\section{SOFTWARE ASSESSMENT HISTORY IN CHINA}

As mentioned in the first paragraph, in the past, nuclear reactor safety analysis software did not need to be reviewed in China because the reactors which were built here in the past were designed by other countries. These reactors had already been built in other countries, and the safety analysis software had already been reviewed by the authority in other countries, so NNSA's position was not to do any other additional independent reviews of the codes. For instance, as AP1000 was designed by Westinghouse and the Nuclear Regulatory Commission (NRC) approved the final design certification for AP1000, the analysis software W-TRAC for AP1000 accident analysis was approved in China.

In 2014, NNSA reviewed an application of using a newly developed fuel management software system for the Qinshan nuclear power plant. This code was developed by the Shanghai Nustar Company independently, and it was the first autonomous software used in Chinese nuclear power plant safety analysis.

In 2015, the Nuclear and Radiation Safety Center began to conduct research on the evaluation methods of autonomous software and conducted detailed research and planning on the licensing conditions, autonomous software evaluation procedures, and document requirements for the application of the software in reactor safety analysis work. The Nuclear and 
Radiation Safety Center also wrote the "safety analysis software assessment methods research report (draft)," which initially determined the implementation plan for autonomous software evaluation.

In 2016, under the framework of the NESTOR software preliminary validation stage, the Nuclear and Radiation Safety Center carried out a series of verification, validation, and thirdparty testing of the NESTOR software. By deeply participating in the software development process as a third party, the Nuclear and Radiation Safety Center had a full understanding of the NESTOR's theoretical model, software framework, and verification status, which laid the foundation for further software safety evaluation.

At the beginning of 2017, NNSA developed a preliminary software evaluation action plan by drawing on the experience of international mature software engineering application certification, and extensively solicited opinions from various domestic nuclear power safety software-developing companies in China on the contents of the plan. In November 2017, NNSA officially published the guidelines "Development and Application of Computer Software for Safety Analysis of Nuclear Power Plant" (China National Nuclear Safety Administration, 2017) and "Implementation measures of Nuclear power plant safety analysis software evaluation." The assessment of safety analysis software for nuclear power plants was officially launched. As of October 2021, 25 pieces of software have been submitted to NNSA.

\section{ASSESSMENT METHOD}

\subsection{Scope}

NNSA only accepts software licensing applications from Chinese companies so far, and the code must be developed for new generation reactors. The software which NNSA accepts can be categorized as follows:

\section{1) Nuclear reactor design code;}

The nuclear reactor design code includes fuel assembly analysis code, reactor core physics analysis code, and spacetime neutron kinetics analysis code. An example of this kind of code is the neurotic cross-section calculation code known as PINE carried out by CGN.

2) Thermal-hydraulics code;

The thermal-hydraulics code is designed to carry out both steady-state and transient analysis, including LOCA analysis codes and sub-channel calculation codes, such as subchannel code CosSub-C developed by SPIC.

3) Containment thermo-hydraulic analysis code;

The containment thermo-hydraulic analysis code is designed to calculate the pressure and temperature behavior of the containment after a LOCA accident.
4) Fuel performance analysis code;

The fuel performance analysis code is designed to simulate the behavior of nuclear fuel during normal operation and an accident situation, such as FUPAC, a fuel performance analysis code developed by NPIC.

5) Radioactive safety analysis code;

The radioactive safety analysis code can simulate radioactive material and radiation, such as the radioactive source term analysis code, radiation-shielding analysis code, and radioactive consequence analysis code.

The severe accident code is not on the list because NNSA holds the opinion that the theory of serious accidents has still not been clarified. Utilizing different calculation models may result in completely opposite calculation results. In addition, there are not enough verification tests that can be used to confirm software for severe accidents.

Table 1 displays the list of software under review and the stage of the review.

\subsection{Evaluation Model}

The evaluation model (EM) is a concept proposed in NRC's regulatory guideline (U.S., 2005). An evaluation model is the calculational framework for evaluating the behavior of the reactor system during a postulated transient or design-based accident. The EM may include one or more computer programs, special models, and all further information needed to apply the calculation framework to a specific event.

China's software evaluation implementation measure has adopted the EM concept. To be more precise, the software evaluation work carried out by NNSA is not only for the software coding itself, but also includes the review of input parameters, model selection, meshing, and other factors. Therefore, software evaluation will only be carried out for the "frozen version" of the software. Applicants are not allowed to make any changes to the software during the code review stage.

\subsection{Procedure}

The software safety evaluation workflow is shown in Figure 1. First, the applicant needs to submit the relevant materials required for the application to NNSA, and then, NNSA will conduct a preliminary review of the software. This preliminary review focuses on whether the format and content of the submitted materials meet the requirements in the "Implementation Measures". After passing the preliminary review, NNSA will officially begin to review the submitted software. After the review, NNSA will formulate a review plan for different pieces of software and initially draw up the evaluation time; however, it should be noted that this evaluation time is not limited for completion but limits the shortest time for evaluation. Generally speaking, for an autonomous piece of software, the shortest evaluation time is 18 months.

After the formal acceptance of the review, the reviewer will begin to review the document report and question the applicant 
TABLE 1 | Software under review and the stage of the review.

\begin{tabular}{|c|c|c|}
\hline Stage of review & Name & Type \\
\hline First round of questioning & Rope & Fuel rod analysis software \\
\hline \multirow[t]{10}{*}{ First round of dialogue } & PALM & Fuel consumption calculation software \\
\hline & POPLAR & One-dimensional Neutronics Program \\
\hline & SuperMC & Monte Carlo calculation simulation software \\
\hline & WILLOW & Core inlet temperature distribution calculation software \\
\hline & GINKGO & System transient analysis software \\
\hline & $\mathrm{BIRCH}$ & Fuel rod temperature analysis software \\
\hline & CPDS & Radioactive system-derived source term calculation program \\
\hline & CPCP & The calculation program of the source term of the primary circuit activation corrosion products \\
\hline & JMCT & 3D Monte Carlo particle transport simulation software \\
\hline & JSNT & Three-dimensional discrete ordinate neutron photon transport software \\
\hline \multirow[t]{2}{*}{ Second round of questioning } & LINDEN & Sub-channel analysis software \\
\hline & CATALPA & Containment Analysis Software \\
\hline \multirow[t]{3}{*}{ Second round of dialogue } & JASMINE & Fuel rod analysis software \\
\hline & CORTH & Sub-channel analysis software \\
\hline & cosSub-C & Sub-channel analysis software \\
\hline Finished & PCM & Core physics analysis software \\
\hline
\end{tabular}

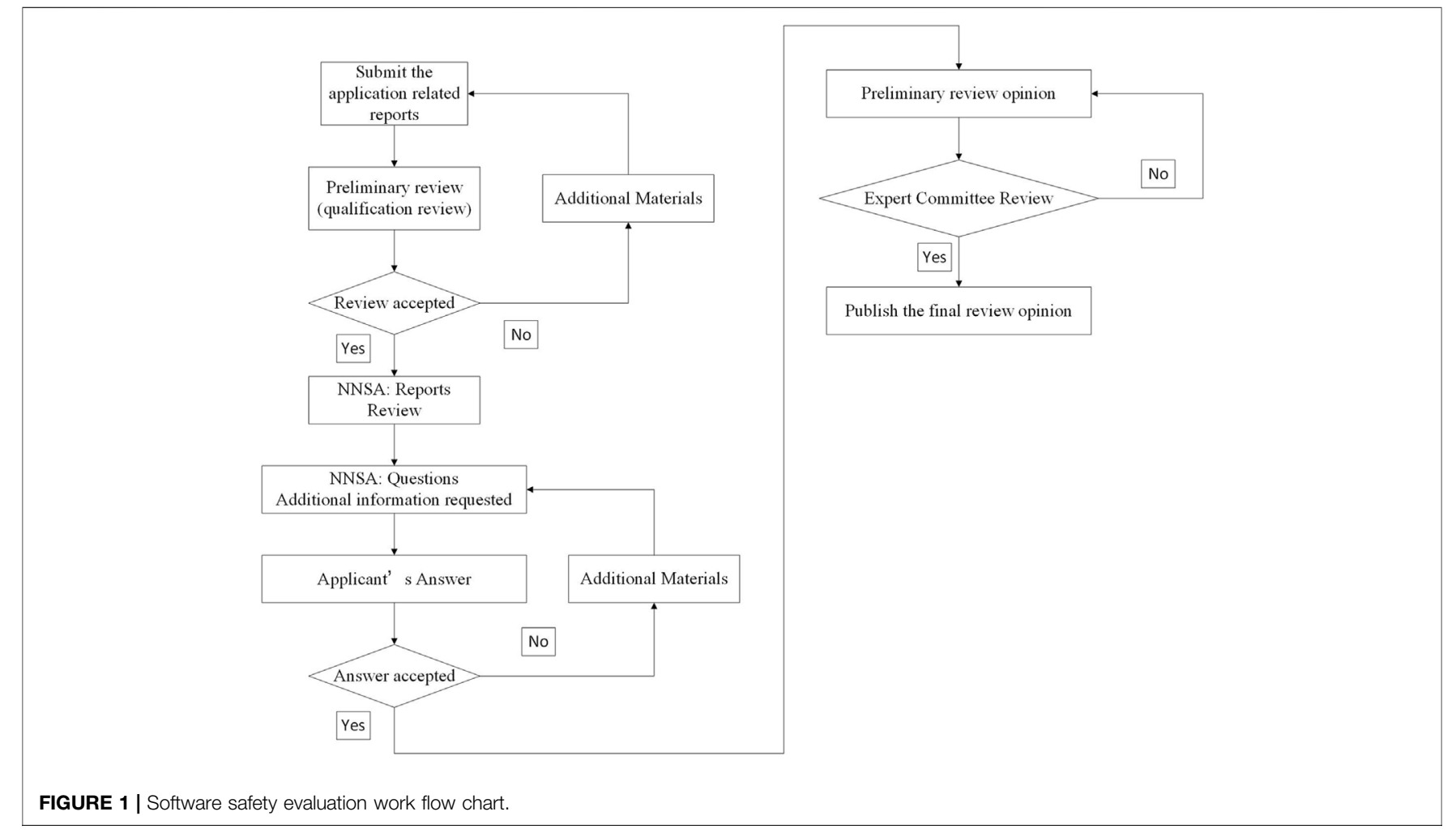

for the problems found in the report, or request that the applicant provide additional information. Generally, there will be at least two rounds of questioning, and after receiving written responses from applicants, a dialogue will be organized to discuss the key issues. Not all issues can be addressed at the dialogue session. On some key issues, where the reviewer and the applicant cannot reach an agreement, this kind of problem will be resolved through more in-depth communication and discussion on the issue by holding a separate thematic meeting.
When all the reviewers' questions are closed, NNSA will ask them to write a software evaluation report. The final report will be submitted to the expert committee, which is composed of software-related experts from various Chinese nuclear research institutions. And those experts with software development experience or software verification experience will be given priority consideration. After being approved by the expert committee, the evaluation report will be published and sent to the applicant. 
Depending on the requirements, applicants should submit six reports, including the software requirement report, V\&V report, software theory report, software quality assurance report, software application object report, and software self-evaluation report. The first four reports have no specific format requirements, but they must include relevant content. The latter two reports, the software self-assessment report and the software application object report, need to be written in the format required by NNSA. The report should contain complete and detailed information about the software. Conceptual or incomplete preliminary information will not be reviewed by NNSA.

Similar to the review of the nuclear power plant document safety analysis report, the software safety evaluation is mainly conducted in the form of a document review. In addition, some audit calculations and check calculations will also be carried out to ensure the authenticity of materials submitted. Check calculation refers to the use of the original input card and program provided by the applicant to reproduce the calculation results of the selected phenomenon and model and to confirm the correctness of the results in the report. Audit calculation means by using the applicant's program, the reviewer chooses some cases or scenarios, builds a new model independently, and then compares the calculation result with the test result. During the process of Audit calculation, the reviewer also often does some sensitivity analyses of the results to deepen their understanding of the software and models. Audit calculation will select models that have a greater impact on the calculation results for verification, and check calculation will randomly select from the calculation examples provided by the applicant.

\subsection{Focus Points of Software Evaluation}

Software evaluation mainly focuses on five aspects.

\section{1) Functional integrity}

The applicator needs to be able to prove that their software has the function of calculating related working conditions. The application scope of different professional software programs is different, and their functions are not completely the same, but the functions of the software must meet all the application requirements.

\section{2) Empirical correlation applicability}

NNSA will focus on whether the theoretical model is correct, whether the source of the theoretical model is reliable, whether the form and coefficients of the theoretical model are suitable for the object calculated by the program, whether the theoretical model has been fully verified, and whether the verification scope covers the parameter range of the simulation object.

Since the evaluation object is the EM, the reviewers will also pay attention to the selection of key parameters of the model, correctness of nodelization, and whether the time step setting is reasonable.

\section{3) Adequacy of validation}

Verification and validation are very important to nuclear safety analysis software, but due to the lack of test data and insufficient validation, validation is often the weakest part in nuclear safety analysis software. Therefore, NNSA will focus on the verification of autonomous software during the review process.

According to the requirements of the software development and application guidelines, the reviewers need to determine whether the validation of the software is sufficient, and they will focus on the process, methods, and logic of the establishment of the confirmation matrix. A complete validation matrix should include the phenomenon to be confirmed, the experiment data used, and the types of experiment data. When identifying the phenomena contained in the validation matrix, each transient/accident that needs to be calculated by the software should be analyzed, so as to evaluate the integrity of the confirmation matrix.

When evaluating the completeness of the phenomena in the confirmation matrix, it is important to sort out models and phenomena which need to be validated completely. It should be noted that code, models, and phenomena which need to be validated should be sorted out from transients or accidents calculated by the program. To validate all the models which the code has is not only unnecessary but also may result in an incomplete validation.

Confirmation data include test data, power plant operation data, domestic and international benchmark questions, analytical solutions, etc., and comparison with similar software is generally not an acceptable basis for confirmation. The reviewer will pay attention to the credibility of the test data and should make a comprehensive judgment based on factors such as the qualifications of the test developer, the year of test development, the completeness of the test report, and the test quality assurance situation.

For the confirmed data from the published article, it should be comprehensively judged based on factors such as the authority of the source of the literature, the year of publication, the completeness of the experimental information in the literature, and the application of other similar pieces of software.

In order to judge whether the test data are available, the reviewer will pay attention to the following aspects:

a) Whether the test objectives and research content are clear, and the test plan is reasonable and feasible;

b) Whether the test party has established a complete quality assurance system and ensured that the test implementation process meets the quality assurance requirements;

c) There should be a similar relationship in physical phenomena between the verification test and the software application target nuclear power plant, and whether the proportional analysis of the test can ensure the correspondence between the test facility and the prototype power plant;

d) The confirmation data in the test should include measurement method information, error information, test conditions, test results, and all relevant parameters necessary for modeling and analysis;

4) Conservatism, sensitivity, and uncertainty

The reviewer will pay attention to the rationality of conservative argumentation, the uncertainty analysis methods, the rationality of the selection of parameters and sensitivity factors, and the uncertainty and the correctness of the sensitivity analysis results. 
Sensitivity analysis can be used as an auxiliary means to evaluate the impact of key parameters on the results. Sensitivity analysis cannot replace uncertainty analysis. The impact of key models, grids, and time steps can be judged by combining the results of sensitivity analysis.

The uncertainty of best estimate software must be evaluated. The applicant can choose different methods to analyze the uncertainty of the software, but the correctness of the method must pass the review.

5) Quality assurance

According to the requirements of software development and application guidelines, the reviewer evaluates the integrity of the quality assurance system, the completeness of the documentation, the compliance of personnel qualifications, etc.

\subsection{Copyrights}

Applicants should have the copyright of the submitted safety analysis software or the certificate of authorization and should also promise to have the legitimate right to use the experimental data used in the validation activity.

\section{ASSESSMENT OF PCM}

The assessment of PCM (Jun and Hao-Liang, 2014) is the first official code assessment project accepted by NNSA, and PCM is the first safety analysis computer code that passed the assessment of NNSA.

NNSA officially accepted the application of assessment of PCM, the nuclear design code package developed by CGN, on February 5, 2018. The PCM code package comprises the neurotic cross-section calculation code PINE and the 3-D nuclear design code COCO. The complete assessment process took 37 months and ended in March 2021. NNSA has issued the accepted application range of the code.

NNSA organized three rounds of questioning (containing 119, 50 , and 29 questions, respectively), two dialogue conferences, two consulting conferences, and one workshop for the assessment of PCM.

The important issues identified during the assessment process are divided into three categories: the correctness of the physical models, the sufficiency of verification and validation, and the rationality of uncertainty analysis. No important issues on physical models were found during the assessment process.

NNSA and the Nuclear and Radiation Safety Center performed an assessment on PCM, which includes physical models, verification and validation, uncertainty analysis, and quality assurance. Several important issues were raised by experts in the process, and the applicant has made substantial supplements and modifications according to the queries of the Nuclear and Radiation Safety Center.

In March 2021, NNSA approved the conclusions of the assessment on PCM, thus finishing the first safety analysis computer code assessment project of China.

\section{CONCLUSION}

This article introduces the safety evaluation work of nuclear power plant analysis software carried out in China, including the scope of software evaluation, evaluation process, and key issues of evaluation concern.

At present, after several years of effort, NNSA's reviewers have accumulated a lot of experience in software validation and confirmation, models, calculation methods, etc., and at the same time have a clearer understanding of the difficulties in software evaluation work. Now in the software evaluation work, there are the following key issues that need to be solved in the application of software engineering in China:

1) Lack of software validation data

Although China has carried out many experiments in the past 10 years and obtained a lot of data, and at the same time, through international cooperation, many international classic data have been obtained as software support. There are still many engineering verification tests that need to be carried out, which is caused by the many unique designs of China's Hualong One and CAP1400.

2) Uncertainty and conservativeness analysis

The uncertainty analysis or conservativeness analysis of the software needs to be fully proved, which requires a rigorous methodology and a lot of confirmation work.

3) Long software evaluation work cycle

Due to lack of experience and technical accumulation, the safety evaluation of PCM software took 3 years. It can be predicted that the evaluation time of thermal hydraulic analysis software will greatly exceed this time.

In order to solve the current lack of data, China is collecting and sorting out power plant data and test data and establishing its own software verification and confirmation database. In the future, new data will be developed and shared, and the credibility of Chinese software will be further enhanced through international cooperation.

\section{AUTHOR CONTRIBUTIONS}

$\mathrm{XG}$ contributed to the conception of the paper and wrote the manuscript; JJ, XH, BJ, XT, and FL contributed significantly to the code assessment project; ChZh contributed significantly to the code assessment methods.

\section{FUNDING}

This research is supported by the National Science and Technology Major Project of China (2019ZX06005001). 


\section{REFERENCES}

Aning, Y., Su, W., Lin, M., Li, Y., and Xu, W. (2019). Typical Benchmarks Verification of Neutron Kinetic Code cosKIND for the Simulation Application of Nuclear Power Plant. Nucl. Sci. Eng. 39 (2), 7.

Brief, C. G. N. (2006). Introduction of China's Improved Pressurized Water Reactor Nuclear Power Plant CPR1000. Mod. Electric Power 023 (005), 36-38. doi:10.3969/j.issn.1007-2322.2006.05.006

Cai, L., Chen, J., and Gao, S. (2016). Development of a 3D Core Calculation Code: $\mathrm{COCO}[J]$. Singapore: Springer.

China National Nuclear Safety Administration (2017). Nuclear Safety Guideline, Development and Application of Computer Software for Safety Analysis of Nuclear Power Plant. Available at: http://www.mee.gov.cn/gkml/sthjbgw/ haq/201712/t20171222_428460.htm

Dong, L., Qing, L., and Zongjian, L. (2017). Development of Nuclear Power Design and Analysis Package NESTOR. China Nucl. Power 010 (004), 532-536. doi:10.12058/zghd.2017.04.532

Ge, W., Yanhua, Y., and Sa, L. (2016). Autonomy of Key Design Software for LargeScale Advanced Pressurized Water Reactor Nuclear Power Plants and Development of COSINE Software Package. China Energy 38 (7), 39-44.

Hao, R. (2015). Breadth First Search Based COSINE Software Code Framework Automation Algorithm.

Hongchun, W. U., Cao, L., Zheng, Y., Li, Y, Zu, T, Liu, Z, et al. (2019). Development and Application of NECP Code Package of Deterministic Nuclear Reactor Physics Code System[J]. Atomic Energy Science and Technology. doi:10.7538/ yzk.2019.53.10.1833

Hu, Y., Chen, Y., Zhang, B., Sun, Y., and Yu, H. (2017). Development and Preliminary Verification of Reactor Shielding Design Code cosSHIELD of COSINE Code Package. Energ. Proced. 127, 87-95. doi:10.1016/ j.egypro.2017.08.113

Jie, W., Tian, W., Su, G., and Qiu, S. Z (2013). Development of TSACO and Application to Chinese HCCB TBM Cooling System[J]. Fusion Eng. Des. 88 (11), 2983-2990. doi:10.1016/j.fusengdes.2013.06.011

Jun, C., Bin, L., Zhaoguo, L., and Liangzhi, C (2017). Development of Homogeneous Subchannel Code of COSINE. Nucl. Sci. Eng. 037 (004), 684-690. 10.1016/j.egypro.2017.08.121 .

Jun, C., and Hao-Liang, L. (2014). Verification of Domestic Core Physical Calculation Software Package PCM in Unit 1 of Ningde Nuclear Power Plant. Chengdu: COPRHY Chengdu, Chinese Nuclear Society.

Ke, W., Jie, J., and Zhihao, R. (2019). Study of CPR1000 Complete Loss of Coolant Flow Accident Based on Sampling Statistical Method. Nucl. Sci. Eng. 039 (004), 581-587. CNKI:SUN:HKXY.0.2019-04-013. doi:10.3969/j.issn.02580918.2019.04.013

Liu, D. (2017). Development of Nuclear Power Design and Analysis Package NESTOR. China Nucl. Power 10 (4). doi:10.12058/zghd.2017.04.532

Ning, B., Zhihao, R., and Mingan, C. (2011). "Research and Development of CGN Thermal-hydraulic Series Software", in Proceedings of the The Fifth Symposium on Reactor Physics and Nuclear Materials and the Second Symposium, Chongqing, China, October 31-November 5, 2021. Nuclear Energy Software Autonomy.

Shiying, W., Chenglong, W., and Guanghui, S. (2018). Development of Analysis Code for Pb-Bi Cooled Direct-Contact-Boiling Water Fast Reactor System[J]. Hedongli Gongcheng/Nuclear Power Eng. 39 (4), 067-070.
Tian, W., Qiu, S., Guo, Y., Su, G, Jia, D, Liu, T, et al. (2007). Development of a Steady thermal-hydraulic Analysis Code for the China Advanced Research Reactor[J]. Front. Energ. Power Eng. China 1 (002), 189-194. doi:10.1007/ s00000-007-0024-8

USNRC (2021). Final Safety Evaluation Report Related to Certification of the AP600 Standard Design. Available at: https://www.nrc.gov/docs/ML9935/ ML993550127.pdf (Accessed 9 Aug 1998).

U.S. (2005). Nuclear Regulatory Commission, REGULATORY GUIDE 1.203, Transient and Accident Analysis Methods.

Wang, C., Yang, S, Peng, S, and Li, G (2018). Automated Validation of CGN Nuclear Software Package PCM. Hedongli Gongcheng/Nuclear Power Eng. 39, 43-46.

Xiao, Y., Hu, L. W., Qiu, S., Zhang, D, Su, G, Tian, W, et al. (2014). Development of a thermal-hydraulic Analysis Code and Transient Analysis for a FHTR[J]. International Conference on Nuclear Engineering. Proceedings: ICONE.

$\mathrm{Xu}, \mathrm{L}$, Cao, L, Zheng, Y, and Wu, H (2017). Development of a New Parallel S-N Code for Neutron-Photon Transport Calculation in 3-D Cylindrical Geometry [J]. Prog. Nucl. engergy 94 (Jan.), 1-21.

Yang, H. Y. (2010). GenerationIVInternational Forum(GIF) RઐD Progress for Sodium Cooled Fast Reactor.

Yu, H., Wang, S, Liu, Z. Q, Wang, C. H, and Chen, Y. X. (2013). Design and Development of Neutron Kinetics Code KIND in COSINE Code Package[J]. Atomic Energy Science and Technology 47(B06), 318-322. doi:10.7538/ yzk.2013.47.S0.0318

Zhang, D. (2016). Generation IV Concepts Handbook of Generation IV Nuclear Reactors, 373-411. doi:10.1016/b978-0-08-100149-3.00014-8

Zhang (1997). SUNF Code for Fast Neutron Data Calculations. China Nuclear Technology Report S3, 24-26.

Zhang, Z., Wu, Z, Sun, Y, and Li, F (2006). Design Aspects of the Chinese Modular High-Temperature Gas-Cooled Reactor HTR-PM. Nucl. Eng. Des. 236, 5485-6490. doi:10.1016/j.nucengdes.2005.11.024

Zhang, Z, Dong, Y, Li, F, Wang, F, Hunag, X, Li, H, et al. (2016). The Shandong Shidao Bay 200 MWe High-Temperature Gas-Cooled Reactor Pebble-Bed Module (HTR-PM) Demonstration Power Plant: An Engineering and Technological Innovation. Engineering. 2(1). doi:10.1016/J.ENG.2016.01.020

Conflict of Interest: The authors declare that the research was conducted in the absence of any commercial or financial relationships that could be construed as a potential conflict of interest.

Publisher's Note: All claims expressed in this article are solely those of the authors and do not necessarily represent those of their affiliated organizations, or those of the publisher, the editors, and the reviewers. Any product that may be evaluated in this article, or claim that may be made by its manufacturer, is not guaranteed or endorsed by the publisher.

Copyright $\odot 2021$ Gao, Jing, Han, Jia, Tian, Liu and Zhang. This is an open-access article distributed under the terms of the Creative Commons Attribution License (CC $B Y)$. The use, distribution or reproduction in other forums is permitted, provided the original author(s) and the copyright owner(s) are credited and that the original publication in this journal is cited, in accordance with accepted academic practice. No use, distribution or reproduction is permitted which does not comply with these terms. 